\title{
Predicting Ecosystem Alliances Using Landscape Theory Shruti Satsangi
}

\author{
"No employer today is independent of those about him." \\ He cannot succeed alone, no matter how great his ability \\ or capital. Business today is more than ever a question of \\ cooperation.
}

Orison Swett Marden (1850-1924)

Writer, physician, and hotelier

\begin{abstract}
Previous articles in the TIM Review have covered various aspects of the concept of business ecosystems, from the types of ecosystems to keystone strategy, to different member roles and value co-creation. While there is no dearth of suggested best practices that organizations should follow as ecosystem members, it can be difficult to apply these insights into actionable steps for them to take. This is especially true when the ecosystem members already have a prior history of cooperation or competition with each other, as opposed to where a new ecosystem is created.
\end{abstract}

Landscape theory, a political science approach to predicting coalition formation and strategic alliances, can be a useful complement to ecosystems studies by providing a tool to evaluate the best possible alliance options for an organization, given information about itself and the other companies in the system. As shown in the case study of mobile device manufacturers choosing platform providers in the mobile ecosystem, this tool is highly flexible and customizable, with more data providing a more accurate view of the alliances in the ecosystem. At the same time, with even basic parameters, companies can glean significant information about which coalitions will best serve their interest and overall standing within the ecosystem.

This article shows the synergies between landscape theory and an ecosystems approach and offers a practical, actionable way in which to analyze individual member benefits.

\section{Introduction}

Increasingly businesses are realizing the realities of the global economy, in which firms must move fast to capture opportunities, revenue, and market share. Rapid innovation cycles are possible today, but only when firms take advantage of complementary assets of other firms and work collaboratively. Stopping to reinvent the wheel can result in firms having to play catch up later, or worse, become irrelevant altogether.
In this new, interdependent business world, Moore's (1993, tinyurl.com/bwjqc3j) seminal work on the structure of businesses as an ecosystem of dependent entities is perhaps the best way to characterize and explain business interactions. This business scenario applies to the mobile industry as well, where the emergence of smartphones over the past six years has resulted in the convergence of traditional and newer players into a collaborative and competitive environment, where firms need to work together to appropriate the most 


\section{Predicting Ecosystem Alliances Using Landscape Theory}

\section{Shruti Satsangi}

value out of their combined market offering as well as compete by differentiating themselves. The mobile business has been repeatedly described in the literature using the framework of business ecosystems theory (Gueguen and Isckia, 2009: tinyurl.com/c6tvff5; Basole, 2009: tinyurl.com/92u58ed; Lin and Ye, 2009: tinyurl.com/ 9zx5se7).

Part of the central premise of an ecosystem is the emergence of a platform on top of which businesses can offer value-added products and services. This type of organization is also seen in the converging mobile ecosystem, where mobile operating system (OS) providers are poised to take the central role of platform providers and serve as a link between the various traditional and newer players in the ecosystem. This central connective role within the industry leads to platform providers being looked at as the keystones of the converging mobile ecosystem, as it is these firms that provide the stable common asset of a mobile OS platform to which other players can link complementary products and value added services (Iansiti and Levien, 2004; tinyurl.com/ bqaol6f). In this way, the actions of a keystone are instrumental in determining the success of every player that is connected to it, and the success of the ecosystem itself.

In order to be successful, keystones must structure their platform such that it can rapidly grow and achieve a large market share and installed base of customers, in other words, become the dominant design. Keeping in mind the interconnected nature of the organizations within an ecosystem, one of the most effective ways to study this growth is using network theory.

Network theory posits that, as a technology platform attracts more users, its perceived value increases and it becomes more attractive to new potential customers. This provides increasing returns as the network increases. This effect is seen time and again within the technology industry and is widely used to explain the emergence of a dominant platform (e.g., Abernathy and Utterback, 1978: tinyurl.com/bt4ql9d; Teece, 1988: tinyurl.com/bpq5nvo; Tegarden et al., 1999: tinyurl.com/c5trt47). In many cases, network effects of a technology are so strong that they can override its deficiencies and disadvantages relative to its competitors.

However, network theory has some limitations. Firstly, for each example of network effects cited in the literature, there are different factors that affect why and how a platform gains traction and attracts users, thereby achieving the dominant status. Deconstructing the suc- cess path often requires deep knowledge of the ecosystem around the platform and industry mechanisms.

A second limitation of network theory is that it is mainly a retrospective analytical tool. It has been used to explain the evolution of a platform or keystone to achieving dominance, but only after the fact. While this offers insights into what factors to consider for future success, these factors change from time to time and industry to industry. Therefore, network theory is not as useful as a predictive device, and when it is used, detailed knowledge and data about the industry is virtually a pre-requisite.

Due to these limitations, network theory does not offer us a full picture of evolving ecosystems, which are still in their nascent stages. This is an apt description of the mobile ecosystem, with several mobile OS providers competing to achieve the status of dominator. Each of these platform providers is trying to attract players offering complementary assets, such as the device manufacturers and applications developers, but offer very different value propositions. As such, there is no clear winner or pre-dominant platform in this ecosystem yet, and it would be interesting to observe how the ecosystem changes over time, until a dominant platform becomes clear.

What would be even more useful, especially for the firms that are looking to make an investment decision to align with one OS platform over the other, would be to be able to predict and analyze which OS they should put their investment dollars in. With the limitations that network theory poses, another approach to solving this problem would be to consider Axelrod and Bennett's (1993; tinyurl.com/ckchctb) landscape theory of aggregation.

Landscape theory is a framework with which Axelrod and Bennett have studied alliance formation. This theory takes a few variables related to the clout of each "actor" in the system (Size) and their tendency to ally with all other actors in the system (this is said to determine their Propensity to work together). Using the Size and Propensity measures, the optimal alliance for the actors in a system can be determined.

The theory has been widely used to predict alliances for World War II, the airline industry, and the setting of UNIX standards, and these retrospective predictions have been corroborated by the actual coalitions that were formed. 


\section{Predicting Ecosystem Alliances Using Landscape Theory}

Shruti Satsangi

Applying this framework to the mobile ecosystem, we can determine which mobile OS platform the mobile device manufacturers, mobile application providers, or mobile network operators should align themselves with in order to appropriate the most value for their business. In this way, landscape theory is a complementary tool to network theory for predicting and understanding business ecosystems and platform success.

\section{Landscape Theory of Aggregation}

Now that it is clear what gaps landscape theory can fill in predicting ecosystem alliance formation, it will useful to understand the landscape theory of aggregation as formulated by Axelrod and Bennett (1993; tinyurl.com/ckchctb). Subsequently, we will discuss how we extended this model by removing some of its limitations.

Aggregation refers to the organization of elements of a system into patterns that tend to put highly compatible elements together and keep less compatible elements apart. Landscape theory predicts how aggregation will lead to alignments or alliances among actors.

For a set of $\mathrm{n}$ actors in any application of the theory, three constructs - size, propensity, and configuration are defined. The size of an actor, $s_{i}>0$, is a reflection of the importance of that actor to others. Size can be measured by demographic, industrial, and economic factors, or any combination of these, depending on what is important in a particular application. The theory states that actors in a system will work to maximize the size of their alliance, because greater size equates to greater access to resources, which can ensure success for the alliance members. In this way, the size measure reflects the concept of cooperation between firms in ecosystems theory.

Another key premise of landscape theory is that two actors have a certain propensity to work together. That propensity will be positive if two actors have a tendency to cooperate with each other, and it will be negative if they do not. In the context of businesses forming alliances, strong competitors will have increased negative propensity to work together. Therefore, propensity is a measure of how willing two firms are to be in the same alliance. In this way, the propensity measure reflects the concept of competition between firms in ecosystems theory. Landscape theory assumes that propensity is a symmetric property of two actors in a system, in other words, actor B will have the same propensity toward actor A that A has towards B.
A configuration is a partition of the actors in a system into one of two groups. This can also be called an alliance structure. For all possible alliance structures, a distance matrix is created, which describes how the actors are arranged in two groups with $\mathrm{Dij}$, the distance between actor $i$ and $j$, being 0 if they are in the same group, and 1 if they are different groups. For example, for four firms, A, B, C, and D, two possible configurations and their respective distance matrices are shown in Figure 1.

By operationalizing size, propensity, and distance with real values, it is possible calculate the utility or payoff function for each actor in each possible alliance structure, as well as the overall "energy" of each structure. The structure that yields the lowest energy value is the optimal alliance configuration per the theory. The formula for energy of the configuration is given below:

$$
E(X)=\sum_{i} \sum_{j} s_{i} s_{j} p_{i j} d_{i j}(X)
$$

The minimization of the energy of a system can be described as the optimal balance of cooperation (measured by size, s) and competition (measured by propensity, p) within the firms in the system, so as to maximize the value they receive by being part of an alliance.

Figure 2 shows the three main constructs of landscape theory: size, propensity, and configuration. Size represents the cooperative need of the firm to create an alliance that will be large and influential enough to ensure success. Propensity represents the competitive nature of the firm and the desire to win over its closest competitors. Configurations represent all the possible ways in which a group of firms can be arranged into two alliances. The configuration yielding the lowest energy is the optimal alliance.

\section{Research Method: Application of Landscape Theory to the Mobile Ecosystem}

As it is presented by Axelrod and Bennett (1993; tinyurl.com/ckchctb), there are some limitations to landscape theory, which have to be dealt with in order to use it to model the mobile ecosystem. The two limitations and their resolutions are as follows:

1. A firm can only belong to one alliance. This limitation means that firms cannot be modeled as being allies of two different mobile platform providers, although this can be a legitimate strategic stance taken by certain firms in the mobile ecosystem. The way in which this 


\section{Predicting Ecosystem Alliances Using Landscape Theory}

\section{Shruti Satsangi}

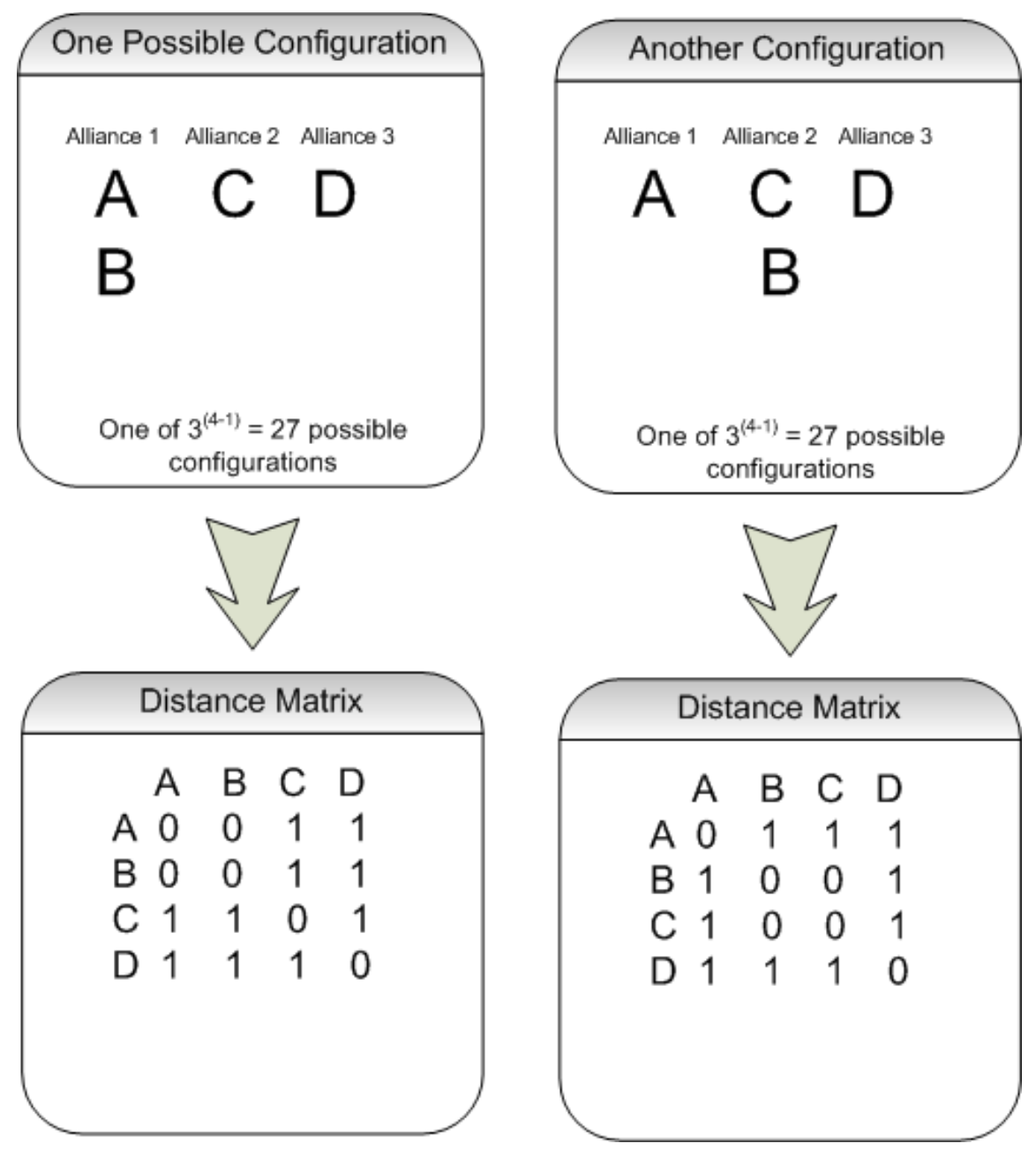

Figure 1. Potential alliance configurations and their corresponding distance matrices for four hypothetical companies

limitation was avoided was to consider only device manufacturers in the sample set. This was based on the network analysis studies done by Basole and Karla (2011; tinyurl.com/8zy7g2t), which show that the mean "degree centrality" (tinyurl.com/8n3poq4) to platform providers (number of connections to platform providers) was under 2 for the device manufacturers while being up to 7 for network operators. By limiting the sample set to device manufacturers, we were able to work within this limitation of the landscape model.

2. Only two alliances are possible with the current theory. This is a limitation when studying the mobile ecosystem because each mobile OS platform provider will have to be modeled as a separate alliance in order to correctly predict which one each device manufacturer will align with. In order to overcome this, the definition of distance was modified so that as long two firms were not in the same alliance, the distance between them was considered to be 1 . This means that Firm A does not care which alliance Firm B is in; as long as Firm B is in a different alliance from $A$, the distance between them will be 1 . If they are in the same alliance, the distance will be 0 .

Once these limitations were taken care of, the next step was to formulate how size and propensity would be measured for the sample set (i.e., mobile device manufacturers and OS platform providers). Fortunately, a similar analysis had previously been done by Axelrod and colleagues (1995; tinyurl.com/8h9qehm) for the Unix workstation industry, which was used for reference. 


\section{Predicting Ecosystem Alliances Using Landscape Theory}

\section{Shruti Satsangi}

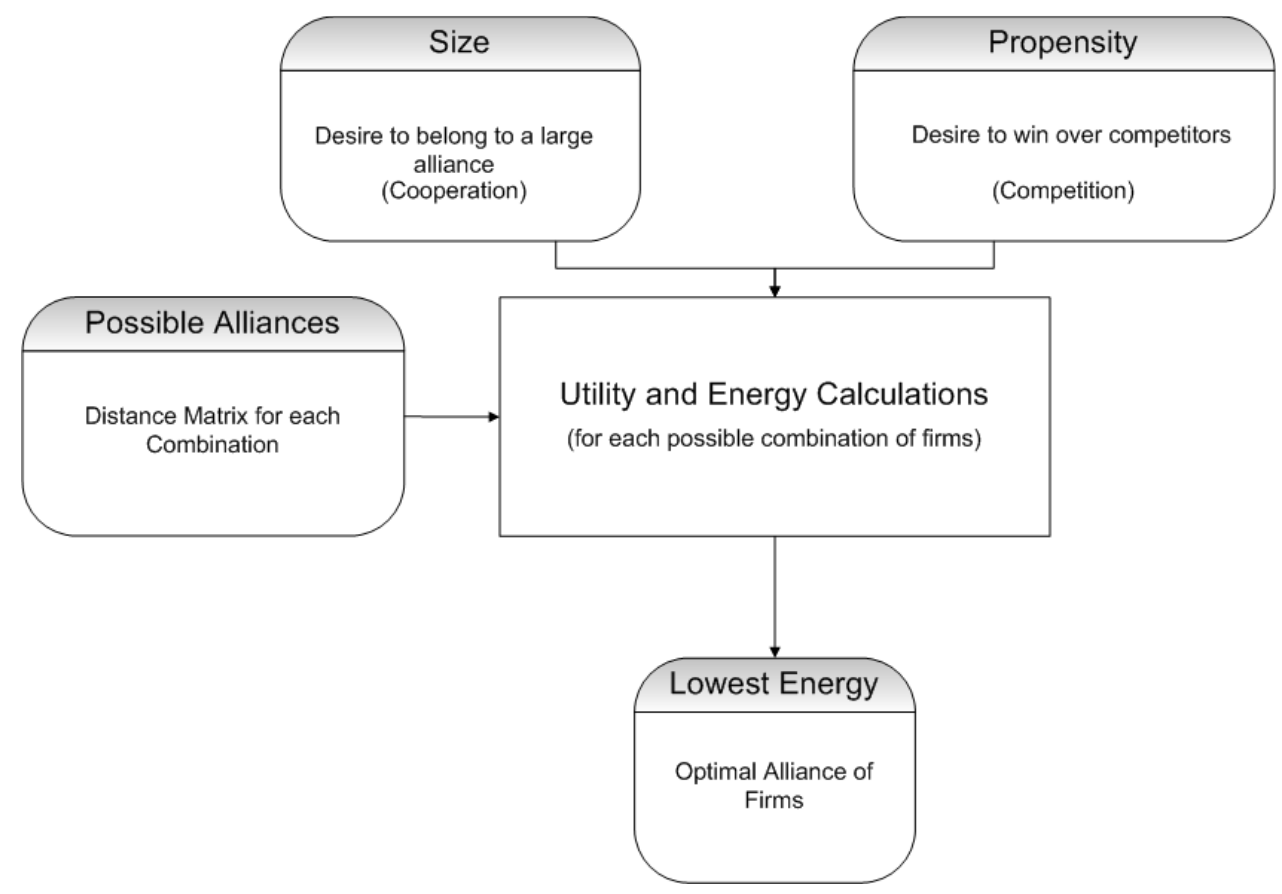

Figure 2. Main constructs of landscape theory

Like the mobile industry, the formation of alliances during the Unix wars (tinyurl.com/ymz326) had the goal of creating standards-setting coalitions. Following the approach used by Axelrod and colleagues in their Unix Wars research paper, the size and propensity equations were formulated as below:

$$
\begin{aligned}
& \text { Size }_{\text {MPP }}=1 / 2(\text { Market Share })+1 / 2(\text { Revenue }) \\
& \text { Size }_{\text {MDM }}=1 / 2(\text { Market Share })+1 / 2(\text { Operating Profit })
\end{aligned}
$$

Market share is a common indicator of firm performance in the mobile industry, making it an easy metric to obtain. Similarly, revenue of a platform provider's (MPP) mobile division indicates the investment capability that a firm may have to launch and establish a mobile OS platform through providing the software development kits (SDKs), support forums, and application stores or marketplaces to fully develop the value network around the platform.

In the case of device manufacturers (MDMs), operating profit is a good indicator of the money a firm has to reinvest into the R\&D activities of developing new mobile phones, which will also involve choosing the mobile OS platform for the handsets.
The propensity equations are as follows:

$$
\begin{aligned}
& \text { PropensityMPP-MPP }=[1-(\alpha+\beta)] \\
& \text { PropensityMPP-MDM }=[1-\alpha]+[\text { Phone Releases on } \\
& \text { MPP(previous year) } / \text { Total Phone Releases }] \\
& \text { PropensityMDM-MDM }=[1-(\alpha+\beta)]
\end{aligned}
$$

Note: $\alpha$ is the basic measure of rivalry, with $\beta$ being an additional rivalry measure, used for close rivals. As defined by the model, $0 \leq \alpha \leq 1$ and $0 \leq \beta \leq 1$, with the base case being $\alpha=1$ and $\beta=1$. By varying the values of $\alpha$ and $\beta$, a sensitivity analysis can be performed on the results of the model.

An important point to note is that propensities between a platform provider and a device manufacturer differ from those between two device manufacturers or two platform providers. Based on Axelrod and colleagues (1995; tinyurl.com/8h9qehm), firms that were both platform providers or were both device manufacturers were modeled as close rivals, while firms in different segments were modeled as distant rivals. Axelrod and colleagues propose that firms serving the same segment will tend to have a greater tendency to compete with 


\section{Predicting Ecosystem Alliances Using Landscape Theory}

\section{Shruti Satsangi}

each other and thus will be more unlikely to ally with one another.

For distant rivals, an additional parameter was considered in the propensity calculation - the actual ties between a platform and device manufacturer, as measured by the phones released on that platform by the manufacturer in the past. This parameter accounts for any additional tendency for a platform provider and device manufacturer to work together, based on past data, making it a good propensity metric.

For this experiment, the sample set was limited to only those platforms that were licensable (i.e., those that allowed adoption by third parties) and to the top $1 \%$ of mobile device manufacturers (so as to select the most important firms to track in the mobile ecosystem). The providers considered were Microsoft (Windows Mobile), Symbian (Symbian OS), and Google (Android). The device manufacturers considered were Nokia, Motorola, Samsung, Sony Ericsson, and LG with the addition of ZTE and Huawei in 2010.
The data gathered to calculate the size and propensity values were all publicly available from the company websites and tax filings. Phone releases by platform and by manufacturer were gathered from phonescoop.com.

Figure 3 summarizes how the landscape model was operationalized for the converging mobile ecosystem.

\section{Results}

Using the above definitions for distance, size, and propensity, energy calculations were made for each possible alliance structure with the firms listed above. The configurations with the lowest energy were the optimal alliances returned by the model.

The results agree well with the visualizations of the actual mobile ecosystem between 2006 and 2010 (Basole and Karla, 2011; tinyurl.com/8zy7g2t). Figure 4 compares the 2010 alliances predicted by the model to the actual alliance visualizations that were carried out by Basole and Karla. This is an apt comparison because the

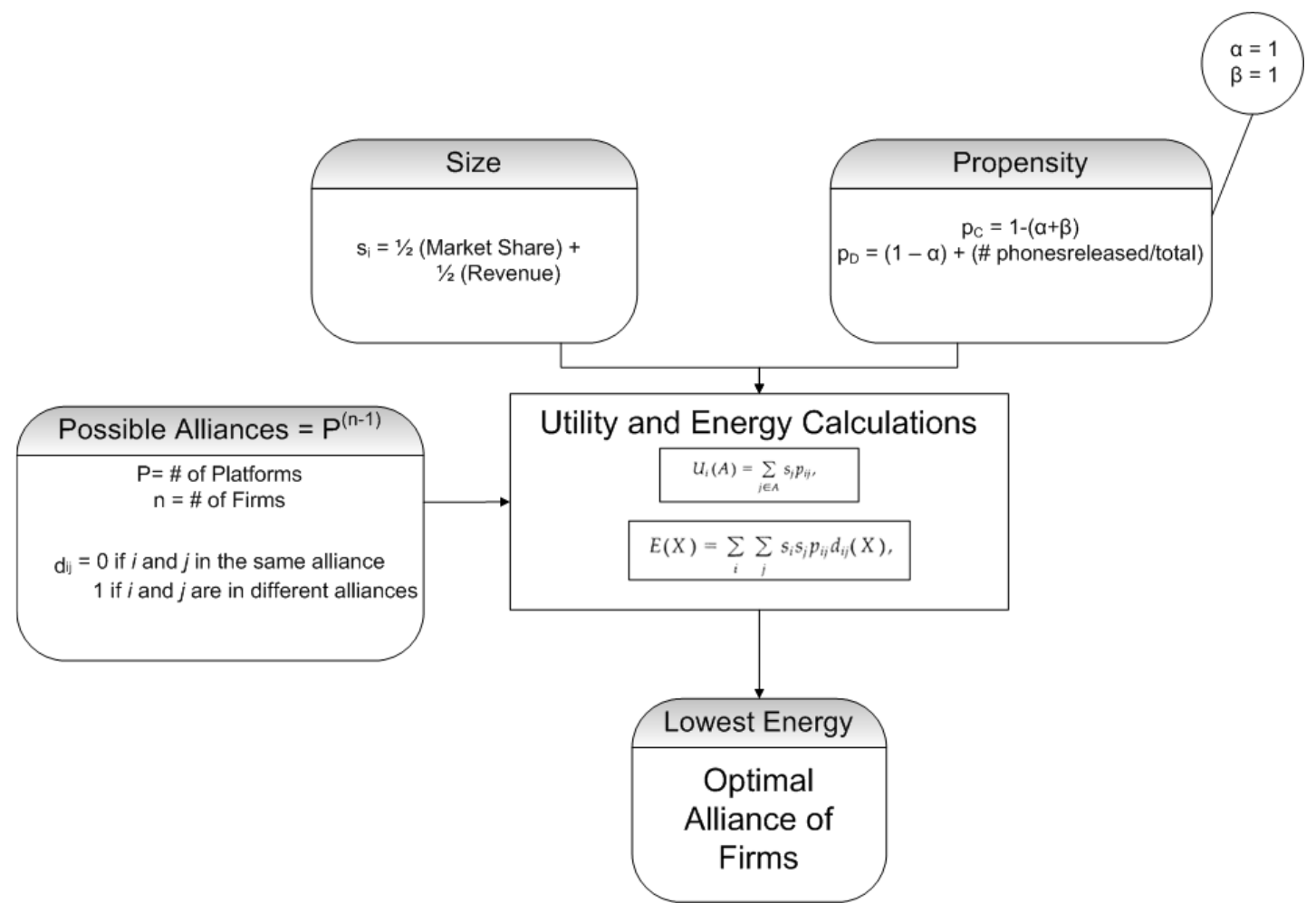

Figure 3. Landscape model for the mobile ecosystems (MDMs and MPPs) 


\section{Predicting Ecosystem Alliances Using Landscape Theory}

\section{Shruti Satsangi}

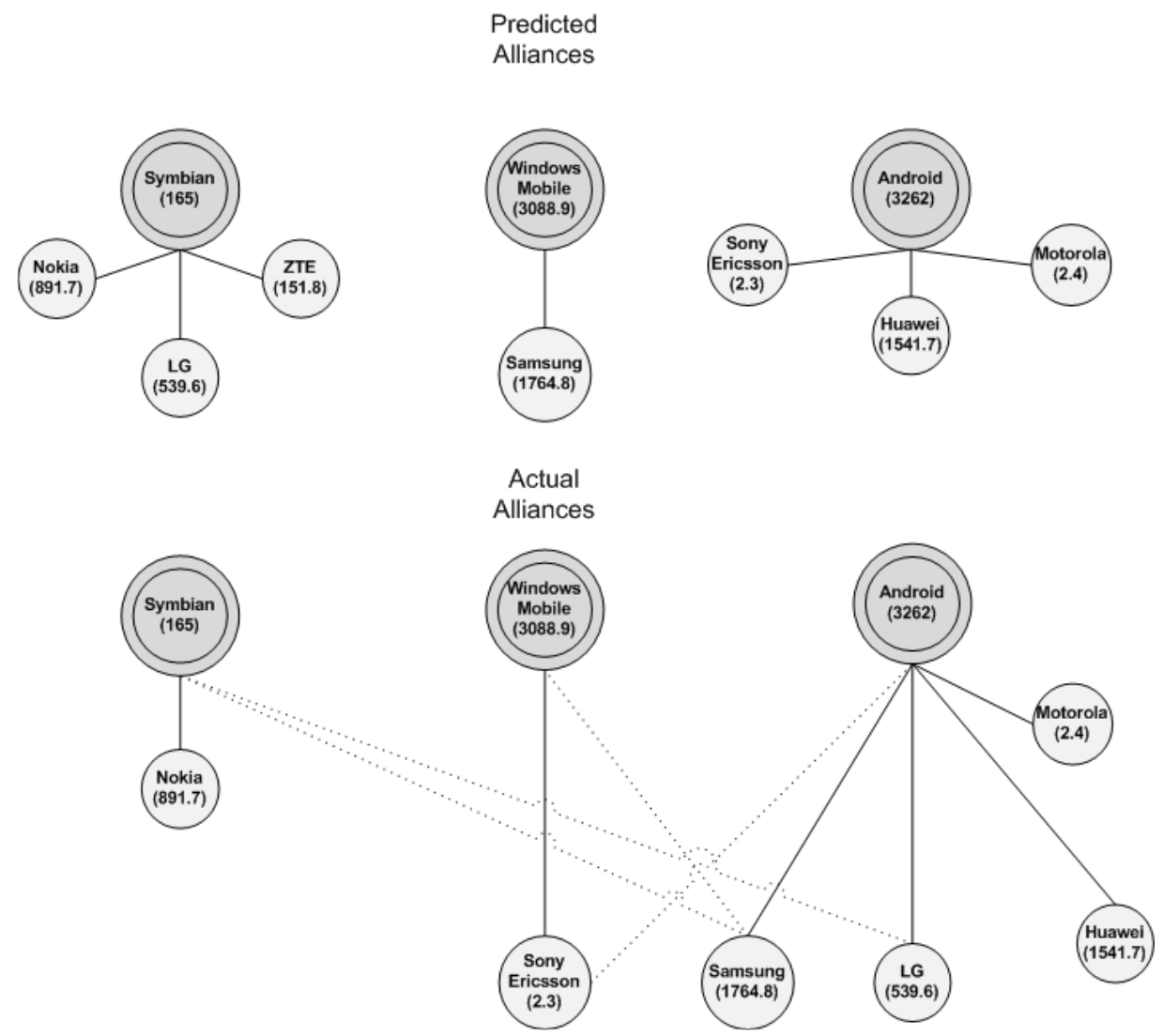

Figure 4. Results predicted by landscape model compared to the actual alliances (2010)

Basole and Karla paper draws the visual representations of the mobile ecosystem using the Kamada-Kawai algorithm (tinyurl.com/casxq6v), which is based on the idea of optimizing a balanced spring system through energy minimization. By using this algorithm, nodes that are close will pull on each other, while those that are distant will push one another apart. In the figure, the platform provider that the manufacturer is closest to (i.e., its strongest ally) is indicated by the solid line while any alliance with other platform providers is indicated by a dotted line. Note that ZTE is not represented in Basole and Karla's paper. Out of the remaining six device manufacturers, we can see that the landscape model results match the actual state of the ecosystem alliances, with the platform choice of Nokia, Motorola, and Huawei predicted correctly. In reality, LG has ties to both Symbian and Android, but the landscape model predicts Symbian as the platform of choice for the firm. A few discrepancies in the alliances are seen for Sony Ericsson and Samsung, with the latter being placed in the Windows alliance while the former is placed in the Android alliance, which are not the results reflected in the visualization. This possibly points to other parameters that are outside the scope of the model that could explain the actual alliance structures that occurred. It also might indicate that the real alliance structures are not the ideal ones for the firms in question.

Overall, the major findings of this research are that:

1. Very basic, universal size and propensity metrics, such as market size and market segment, are well supported by the visualizations of the real state of the ecosystem between 2006 and 2010.

2. At times, the model returns optimal alliances contrary to the actual alliances at that time, but those results make more sense when analyzed in retrospect. For example, in reality, Sony Ericsson stuck to the Symbian platform for longer than was advisable based on the predictions of the landscape model. This delay could account for the significant loss of market share experienced by the firm. Based on the results of the model, Sony Ericsson should have committed to Android 


\section{Predicting Ecosystem Alliances Using Landscape Theory}

\section{Shruti Satsangi}

much earlier. In addition to this, sometimes the tool shows the basis for alliance decisions that firms took after 2010. For example, the acquisition of Motorola Mobility by Google is supported by the alliances predicted in 2010 by the model. It clearly shows that Motorola will choose Android as its OS platform. This suggests that the model has some value as a predictive tool.

\section{Conclusions}

As shown, not only does landscape theory provide meaningful results as a predictive and analytical model for device manufacturers to strategically pick mobile OS platforms for their future phone offerings, but it shows great promise in its extensibility to various other players in the mobile ecosystem, as well as business ecosystems in general.

Through this research, landscape theory also shows itself to be a useful framework to employ where network theory has its limitations, namely, the lack of predictive ability for a still-evolving ecosystem and the requirement of significant industry knowledge to be a useful explanatory tool. With the landscape model, very basic, universal and publicly available business metrics such as market share and revenue can be used to gain substantial insight into how firms should align themselves to maximize their future success. In this way, the model is complementary to network theory in the understanding of ecosystem evolution.

Furthermore, the extensibility and flexibility of the model is such that adding industry specific knowledge (by refining the propensity definition accordingly) serves to further improve its efficacy. In this way, landscape theory can be combined with network theory, measuring the network effects of different industry factors by modeling them as part of the size and propensity constructs, as appropriate.

By clearly showing the parallels between business ecosystems research and landscape theory through the study of alliance formation in the mobile industry, this research shows the harmony between these two research streams and opens up new avenues for further research involving both these theoretical frameworks. It is hoped that this is of interest to researchers and scholars of both these fields and that future research be undertaken to further explore links between them and their applicability to other industries besides mobile.
In industry terms, this research may be of great relevance to strategic decision makers within private (businesses) and public organizations (government) seeking to better understand and plan the alliances into which they enter and also to understand the overall shape of the ecosystem to which they belong. The landscape theory model applied in the context of a business ecosystem is practical way in which they can achieve the goal of aligning themselves with the correct platform and partners to achieve success.

\section{Recommended Reading and Resources}

1. Ken Binmore's review of Robert Axelrod's book, The Complexity of Cooperation: Agent-Based Models of Competition and Collaboration, provides helpful background information on landscape theory: tinyurl.com/8ccrwmw

2. Complete results from this research, including links to the raw data and code written to perform the calculations, are available here:

shrutisatsangi.com/research

\section{About the Author}

Shruti Satsangi is a recent graduate of the Technology Innovation Management program at Carleton University, in Ottawa, Canada, where she researched coalition and competition within business ecosystems. Her Master's thesis focused on implementing landscape theory to better explain coalition formation within the mobile ecosystem. Ms. Satsangi received a Bachelor's in Computer Engineering from the University of Waterloo with specializations in Management Science and Telecommunications. She has extensive experience in the development of large, carrier-grade $4 \mathrm{G}$ mobile communication systems. Shruti is currently serving as a committee member for the Innovation and Entrepreneurship track at the Grace Hopper Conference 2012. She is also a member of CU-Women in Science and Engineering, IEEE WiE, and the IEEE Communications Society and an occasional guest blogger for the Anita Borg Institute.

Citation: Satsangi, S. 2012. Predicting Ecosystem

Alliances Using Landscape Theory. Technology

Innovation Management Review. August 2012: 31-38. (cc) BY 